\title{
Biopelícula a partir de almidón de banano verde (Musa paradisiaca) y mandioca (Manihot esculenta)
}

\section{Biofilm from green banana starch (Musa paradisiaca) and cassava (Manihot esculenta)}

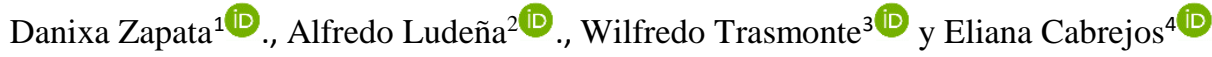

\begin{abstract}
RESUMEN
El objetivo de esta investigación fue evaluar la influencia de diferentes concentraciones y tipo de almidón sobre las propiedades de las películas, las mismas que fueron obtenidos por el método de Casting a partir de mezclas de almidón de banano verde y mandioca a concentraciones de $3 \%$ y $4 \%$ con quitosano comercial al $1 \%$, combinados con glicerina como agente plastificante y gel de sábila como agente antifúngico a concentración del $5 \%$. Las películas biodegradables formuladas se evaluaron en propiedades fisicoquímicas, mecánicas y biodegradables, aplicando diseño en bloques completamente aleatorizados. Se determinó que el espesor de las películas biodegradables aumenta de manera significativa, al aumentar la concentración de almidón, influyendo en la permeabilidad al vapor de agua, en la solubilidad y en el esfuerzo de tracción en el corte, pero no en la humedad; las películas biodegradables formuladas con almidón y quitosano tienen mejores propiedades mecánicas y biodegradables que las elaboradas solo con almidón. Así mismo, las propiedades de las películas biodegradables están influenciadas por la concentración de almidón y en menor medida por la fuente de almidón.
\end{abstract}

Palabras clave: Películas biodegradables, almidón, propiedades físicoquímicas, propiedades mecánicas, biodegradabilidad.

\begin{abstract}
The objective of this research was to evaluate the influence of different concentrations and type of starch on the properties of the films, which were obtained by the Casting method from mixtures of green banana and cassava starch at concentrations of $3 \%$ and $4 \%$ with commercial chitosan at $1 \%$, combined with glycerin as plasticizing agent and aloe vera gel as an antifungal agent at a concentration of 5\%. The formulated biodegradable films were evaluated in physicochemical, mechanical and biodegradable properties, applying completely randomized block design. It was determined that the thickness of biodegradable films increases significantly, when starch concentration increases, influencing water vapor permeability, solubility and traction effort in cutting, but not humidity; biodegradable films formulated with starch and chitosan have better mechanical and biodegradable properties than those elaborated only with starch. Also, the properties of biodegradable films are influenced by the concentration of starch and to a lesser extent by the source of starch.
\end{abstract}

Keywords: Biodegradable films, Starch concentration, Physicochemical properties, Mechanical properties, Biodegradability.

DOI: https://doi.org/10.37787/pakamuros-unj.v8i4.146

Recibido: 08/11/2020. Aceptado: 29/11/2020

* Autor para correspondencia

1. Universidad Nacional de Piura, Perú. Email: danixazapatac@gmail.com; ludesalf@ hotmail.com

2. Universidad Nacional San Cristóbal de Huamanga, Perú. Email: wilfredo.trasmonte@unsch.edu.pe

3. Universidad Nacional de Jaén, Perú. Email: elianacabrejos@gmail.com.pe 


\section{INTRODUCCIÓN}

Es importante el cuidado del medio ambiente y el pensar en el uso de empaques biodegradables contribuye a reducir la contaminación ambiental y a reducir el uso de empaques sintéticos que generan contaminación.

Los plásticos convencionales, producidos a partir de derivados del petróleo, originan enormes problemas de contaminación ambiental, permaneciendo como contaminantes durante períodos prolongados de tiempo. El plástico es la tercera aplicación del petróleo más usada en el mundo, y al año consumimos 300 millones de toneladas y como consecuencia se lanzan a la atmósfera toneladas de dióxido de carbono. Al adquirir productos con envases hechos de plástico y desechar estos, inconscientemente estamos contaminando al planeta (Vázquez, Beltrán, Espinosa y Velasco, 2018).

La bioingeniería ha generado la creación de "bioplásticos" o "plásticos verdes" que son 100\% degradables, su tiempo de degradación no tarda más de un año, están hechas a partir de resinas vegetales, almidones, contaminan 85 \% menos que el plástico convencional (Castillo, Escobar y Fernández, 2015). Las biopelículas o películas biodegadables, fabricadas a partir de almidón comparten las características con los elaborados a través de derivados de petróleo (Alves et al.,2007). La mayor diferencia entre el plástico que actualmente se fabrica y el producido con base en almidón, se da porque el segundo es completamente biodegradable y no tóxico, una vez degradado puede usarse incluso como material de compostaje (abono). Esta ventaja proviene, precisamente, en el origen vegetal de la materia prima, lo que reduce nuestra dependencia del petróleo, que desde décadas domina nuestra sociedad.

Con el desarrollo de esta investigación se busca reducir la contaminación con la elaboración de un producto que reemplace al plástico, de tal forma que ayude tanto al medio ambiente como a los seres vivos que habitan en él.

Durante la investigación se realizó la evaluación de las características de las biopelículas formuladas a partir de almidón de banano verde (Musa paradisiaca) y mandioca (Manihot esculenta) con adición de gel de sábila (Aloe vera), logrando caracterizar los almidones utilizando como matriz del bioplástico.

\section{MATERIALES Y MÉTODOS}

La investigación tuvo lugar en el laboratorio de agroindustrias de la Facultad de Ingeniería Industrial de la Universidad Nacional de Piura y en el laboratorio forestal de la Universidad Nacional de Tumbes durante el año 2019.

Las materias primas empleadas fueron el banano verde y mandioca de segunda y tercera categoría extraídos de la producción de áreas agrícolas del Empreñadero de Santa Cruz, Región de Piura, y la sábila en las áreas verdes de la Universidad Nacional de Tumbes, glicerina, quitosano, agua destilada. 
Se realizó la extracción del almidón por el método húmedo, se extrajo el gel de la sábila, se caracterizaron los almidones determinando los porcentajes de humedad, ceniza, lípidos de ambos almidones y se determinó el porcentaje de amilosa y amilopectina por espectrofotometría; obteniéndose las películas biodegradables por el método Casting (vertido en placas), combinando los almidones al $3 \%$ y $4 \%$ (p/v) con agua destilada como agente base estructural, glicerina al 1,5\% (p/v) y quitosano al $1 \%(\mathrm{p} / \mathrm{v})$ como agentes plastificantes, gel de sábila al $5 \%(\mathrm{p} / \mathrm{v})$ como un agente anti fúngico en el orden indicado; se determinó las propiedades físico-mecánicas, y biodegradable de las películas obtenidas.

Los tratamientos evaluados de las biopelículas al $3 \%$ y $4 \%$ de banano verde y mandioca fueron: T1: 70 $\%, 20 \%$ y $10 \%$ de soluciones de almidón, gel de sábila y glicerina; T2: $60 \%, 25 \%$ y $15 \%$ de soluciones de almidón, gel de sábila y glicerina; T3: $60 \%$, $30 \%$ y $10 \%$ de soluciones de almidón, gel de sábila y glicerina; T4: $40 \%, 10 \%, 10 \%, 40 \%$ de soluciones de almidón, gel de sábila, glicerina y quitosano; T5: $30 \%, 10 \%, 10 \%, 50 \%$ de soluciones de almidón, gel de sábila, glicerina y quitosano.

Se determinó las propiedades físicas de las películas biodegradables como la medición del espesor humedad, solubilidad y permeabilidad al vapor de agua (Norma ASTM E96M/E96-05); las propiedades mecánicas para medir el esfuerzo de tracción o tensión de corte, porcentaje de elongación (Norma ASTM D882, 2000) y las propiedades de biodegradación aeróbicas y anaeróbicas en el suelo a $12 \mathrm{~cm}$ de profundidad (Rutiaga, 2002).

\section{RESULTADOS}

Los resultados del análisis químico de los almidones utilizados se describen en la Tabla 1.

Tabla 1. Composición química de los almidones.

\begin{tabular}{lccc}
\hline \multicolumn{1}{c}{ Muestras } & Humedad* $(\boldsymbol{\%})$ & Cenizas* $(\%)^{*}$ & Lípidos* $(\%)^{*}$ \\
\hline Almidón de Banano verde & $15.61 \pm 0.31$ & $0.26 \pm 0.05$ & $0.43 \pm 0.08$ \\
Almidón de Mandioca & $9.53 \pm 0.11$ & $0.07 \pm 0.04$ & $0.39 \pm 0.11$ \\
\hline
\end{tabular}

*Todos los resultados son promedio de dos determinaciones. Media \pm Desviación estándar.

De la tabla anterior, a mayor contenido de humedad presente en el almidón, mayor es el porcentaje de cenizas. La desventaja está en que un almidón muy húmedo se atribuye principalmente a su carácter altamente hidrófilo de los polímeros de almidón de banano verde.

El banano verde obtuvo el mayor porcentaje de humedad de $15.61 \%$ en comparación al almidón de mandioca de $9.53 \%$.

Respecto al contenido de cenizas para el almidón de banano verde se encontró el $0.26 \%$. Para el almidón de mandioca se obtuvo el $0.07 \%$ cenizas.

El contenido de lípidos encontrado en estudio de investigación osciló a $0.43 \%$ para la muestra de almidón de banano verde, mayor comparado con el almidón de mandioca. 
Tabla 2. Resultados del contenido de Amilosa y Amilopectina

\begin{tabular}{lccc}
\hline \multicolumn{1}{c}{ Muestras } & Absorbancia & $\begin{array}{c}\text { Contenido de } \\
\text { amilosa (\%) }\end{array}$ & $\begin{array}{c}\text { Contenido de } \\
\text { amilopectina (\%) }\end{array}$ \\
\hline Almidón de Banano Verde & $0.557 \pm 0.006$ & $20.43 \pm 0.05$ & $79.57 \pm 0.05$ \\
Almidón de Mandioca & $0.455 \pm 0.001$ & $14.36 \pm 0.04$ & $85.64 \pm 0.04$ \\
\hline
\end{tabular}

Las muestras del almidón de banano verde y mandioca están dadas entre el 15 - 30\%, la diferencia 70 $85 \%$ corresponde a amilopectina, clasificándolas como almidones normales.

Tabla 3. Prueba de Tukey para la humedad en películas de almidón de banano verde al $3 \%$ y $4 \%$

\begin{tabular}{lcccc}
\hline \multirow{2}{*}{ Tratamiento } & \multicolumn{2}{c}{ Almidón de banano verde al 3\% } & \multicolumn{2}{c}{ Almidón de banano verde al 4\% } \\
\cline { 2 - 5 } & $\begin{array}{c}\text { Humedad Promedio } \\
(\boldsymbol{\%})\end{array}$ & $\begin{array}{c}\text { Tukey } \\
\mathbf{\alpha}=\mathbf{0 . 0 5}\end{array}$ & $\begin{array}{c}\text { Humedad Promedio } \\
(\boldsymbol{\%})\end{array}$ & $\begin{array}{c}\text { Tukey } \\
\boldsymbol{\alpha}=\mathbf{0 . 0 5}\end{array}$ \\
\hline T2 & 4.79 & $\mathrm{a}$ & 2.66 & $\mathrm{~b}$ \\
$\mathrm{~T} 4$ & $\mathrm{ab}$ & 3.63 & $\mathrm{a}$ \\
$\mathrm{T} 1$ & 4.08 & $\mathrm{ab}$ & 3.42 & $\mathrm{a}$ \\
$\mathrm{T} 5$ & $\mathrm{~b}$ & 1.65 & $\mathrm{~b}$ \\
$\mathrm{~T} 3$ & 3.30 & $\mathrm{~b}$ & 1.37 & $\mathrm{~b}$ \\
\hline
\end{tabular}

La prueba Tukey muestra que el tratamiento $\mathrm{T} 2$ es significativamente diferente a $\mathrm{T} 5$ y $\mathrm{T} 3$ pero significativamente igual a T5 ocupando el primer orden con humedad promedio de $4.79 \%, 4.08 \%$ y $3.30 \%$ respectivamente; asimismo, se observa que $\mathrm{T} 4$ es significativamente diferente a $\mathrm{T} 2$, pero no existe diferencia significativa con T1, T5 y T3, ocupando estos el segundo orden.

La Tabla 3, muestra que el tratamiento $\mathrm{T} 4$ es significativamente diferente a $\mathrm{T} 5$ y $\mathrm{T} 3$ pero significativamente igual a T1 y T2 ocupando el primer orden con humedad promedio de $3.63 \%, 3.42 \%$ y $2.66 \%$ respectivamente; asimismo, se observa que $\mathrm{T} 2$ es significativamente diferente a $\mathrm{T} 4$ y $\mathrm{T} 1$ pero no existe diferencia significativa en $\mathrm{T} 5$ y T3, ocupando estos el segundo orden.

En cuanto a las películas formuladas a partir de banano verde, se registraron valores de contenido de humedad más altos cuando la concentración de almidón es menor, es decir, tienen mayor porcentaje de humedad las películas de almidón de banano verde al 3 \% y menor en las películas con una concentración de almidón de $4 \%$. 
Tabla 4. Prueba de Tukey para la humedad en películas de almidón de mandioca al 3\%. Y $4 \%$

\begin{tabular}{lcccc}
\hline \multirow{2}{*}{ Tratamiento } & \multicolumn{2}{c}{ Almidón de mandioca al 3 \% } & \multicolumn{2}{c}{ Almidón de mandioca al 4 \% } \\
\cline { 2 - 5 } & $\begin{array}{c}\text { Humedad Promedio } \\
(\boldsymbol{\%})\end{array}$ & $\begin{array}{c}\text { Tukey } \\
\boldsymbol{\alpha}=\mathbf{0 . 0 5}\end{array}$ & $\begin{array}{c}\text { Humedad Promedio } \\
(\boldsymbol{\%})\end{array}$ & $\begin{array}{c}\text { Tukey } \\
\boldsymbol{\alpha}=\mathbf{0 . 0 5}\end{array}$ \\
\hline T5 & 2.47 & $\mathrm{a}$ & 5.31 & $\mathrm{a}$ \\
T2 & 2.26 & $\mathrm{ab}$ & 1.33 & $\mathrm{c}$ \\
T3 & 1.56 & $\mathrm{abc}$ & 1.48 & $\mathrm{c}$ \\
T1 & 1.20 & $\mathrm{bc}$ & 0.54 & $\mathrm{~d}$ \\
T4 & 0.51 & $\mathrm{c}$ & 3.93 & $\mathrm{~b}$ \\
\hline
\end{tabular}

La prueba Tukey indica que el tratamiento T5 es significativamente diferente a T1 y T4 pero significativamente igual a T2 y T3 ocupando el primer orden con humedad promedio de $2.47 \%, 2.26 \%$ y $1.56 \%$ respectivamente.

La prueba Tukey para el almidón de mandioca al $4 \%$ indicó que el tratamiento T5 es significativamente diferente con todos los tratamientos al igual que el T4.

Tabla 5. Prueba de Tukey para la solubilidad de películas de almidón de banano verde al $3 \%$ y $4 \%$

\begin{tabular}{lcccc}
\hline \multirow{2}{*}{ Tratamiento } & \multicolumn{2}{c}{ Almidón de banano verde al 3\% } & \multicolumn{2}{c}{ Almidón de banano verde al 4\% } \\
\cline { 2 - 5 } & $\begin{array}{c}\text { Solubilidad } \\
\text { Promedio }(\%)\end{array}$ & $\begin{array}{c}\text { Tukey } \\
\boldsymbol{\alpha}=\mathbf{0 . 0 5}\end{array}$ & $\begin{array}{c}\text { Solubilidad } \\
\text { Promedio }(\%)\end{array}$ & $\begin{array}{c}\text { Tukey } \\
\boldsymbol{\alpha}=\mathbf{0 . 0 5}\end{array}$ \\
\hline T2 & 26.59 & $\mathrm{a}$ & 18.09 & $\mathrm{bc}$ \\
T5 & 23.30 & $\mathrm{ab}$ & 23.33 & $\mathrm{a}$ \\
T4 & 20.17 & bc & 20.20 & $\mathrm{ab}$ \\
T3 & 19.45 & bc & 14.76 & $\mathrm{c}$ \\
T1 & $16 . .32$ & $\mathrm{c}$ & 16.52 & $\mathrm{bc}$ \\
\hline
\end{tabular}

La prueba Tukey indicó que el tratamiento T2 es significativamente diferente a T4, T3 y T1 pero significativamente igual a T5 ocupando el primer orden con solubilidad promedio de $26.59 \%$ y $23.30 \%$ respectivamente

Los datos obtenidos para el almidón de banano verde al 4\% indica que el tratamiento T5 es significativamente diferente a T3, T1 y T2 pero significativamente igual a T4 ocupando el primer orden con solubilidad promedio de $23.33 \%$ y $20.20 \%$ respectivamente.

Tabla 6. Prueba de Tukey para la solubilidad de películas de almidón de mandioca al 3\% y 4\%.

\begin{tabular}{lcccc}
\hline \multirow{2}{*}{ Tratamiento } & \multicolumn{2}{c}{ Almidón de mandioca al 3\% } & \multicolumn{2}{c}{ Almidón de mandioca al 4\% } \\
\cline { 2 - 5 } & $\begin{array}{c}\text { Solubilidad } \\
\text { Promedio }(\%)\end{array}$ & $\begin{array}{c}\text { Tukey } \\
\boldsymbol{\alpha}=\mathbf{0 . 0 5}\end{array}$ & $\begin{array}{c}\text { Solubilidad } \\
\text { Promedio }(\%)\end{array}$ & $\begin{array}{c}\text { Tukey } \\
\boldsymbol{\alpha}=\mathbf{0 . 0 5}\end{array}$ \\
\hline T3 & 32.36 & $\mathrm{a}$ & 17.30 & $\mathrm{~b}$ \\
T5 & 25.15 & $\mathrm{ab}$ & 17.82 & $\mathrm{~b}$ \\
T4 & 21.37 & $\mathrm{ab}$ & 19.41 & $\mathrm{ab}$ \\
T1 & 14.25 & $\mathrm{~b}$ & 17.30 & $\mathrm{~b}$ \\
T2 & 13.62 & $\mathrm{~b}$ & 22.73 & $\mathrm{a}$ \\
\hline
\end{tabular}


La prueba Tukey muestra que el tratamiento $\mathrm{T} 3$ es significativamente diferente a $\mathrm{T} 1$ y $\mathrm{T} 2$, pero significativamente igual a T5 y T4 ocupando el primer orden con solubilidad promedio de 32.36 \%, 25.15 \% y $21.37 \%$ respectivamente. Para el almidón de mandioca verde al 4\% indica que el tratamiento T2 es significativamente diferente a T5, T3 y T1 pero significativamente igual a T4 ocupando el primer orden con solubilidad promedio de $22.73 \%$ y $19.41 \%$ respectivamente; asimismo, se puede apreciar que la permeabilidad al vapor de agua en el almidón de banano verde $3 \%$ oscila entre 0.00058 y 0.00034 $\mathrm{gmm} / \mathrm{hPam}^{2}$. Correspondiendo éstas a T2 y T3 respectivamente, siendo indicadas como el valor de mayor y menor permeabilidad.

Tabla 7. Prueba Tukey para la permeabilidad de vapor de agua (PVA) en películas de almidón de banano verde al 3\% y $4 \%$.

\begin{tabular}{lcccc}
\hline \multirow{2}{*}{ Tratamiento } & \multicolumn{2}{c}{ Almidón de banano verde al 3\% } & \multicolumn{2}{c}{ Almidón de banano verde al 4\% } \\
\cline { 2 - 5 } & $\begin{array}{l}\text { Permeabilidad } \\
\text { Promedio (\%) }\end{array}$ & $\begin{array}{c}\text { Tukey } \\
\boldsymbol{\alpha}=\mathbf{0 . 0 5}\end{array}$ & $\begin{array}{c}\text { Permeabilidad } \\
\text { Promedio (\%) }\end{array}$ & $\begin{array}{c}\text { Tukey } \\
\boldsymbol{\alpha}=\mathbf{0 . 0 5}\end{array}$ \\
\hline T2 & 0.00058 & $\mathrm{a}$ & 0.00058 & $\mathrm{Ab}$ \\
T5 & 0.00052 & $\mathrm{ab}$ & 0.00052 & $\mathrm{Ab}$ \\
$\mathrm{T} 1$ & 0.00049 & $\mathrm{abc}$ & 0.00071 & $\mathrm{~A}$ \\
T4 & 0.00039 & $\mathrm{bc}$ & 0.00044 & $\mathrm{~B}$ \\
T3 & 0.00034 & $\mathrm{c}$ & 0.00040 & $\mathrm{~B}$
\end{tabular}

La prueba de Tukey indicó que el tratamiento T2 es significativamente diferente a T4 y T3 pero significativamente igual a T5 y T1 ocupando el primer orden con permeabilidad al vapor de agua promedio de $0.00058 \%, 0.00052 \%$ y $0.00049 \%$ respectivamente; asimismo, se observa que el tratamiento T5 es significativamente diferente a T2 y T3 pero no existe diferencia significativa en T1 y T4, ocupando estos el segundo orden. Por consiguiente, se aprecia que el tratamiento T1 es significativamente igual a T4 y T3 pero no a T2 y T5 ocupando el tercer orden. Para la muestra de almidón de banano verde al 4\% muestra que el tratamiento T1 es significativamente diferente a T4 y T3 pero significativamente igual a T2 y T5 ocupando el primer orden con permeabilidad al vapor de agua promedio de $0.00071 \%, 0.00058 \%$ y $0.00052 \%$ respectivamente; asimismo, se observa que el tratamiento $\mathrm{T} 2$ es significativamente diferente a $\mathrm{T} 1$ pero no existe diferencia significativa en los tratamientos T5, T4 y T3, ocupando estos el segundo orden.

Tabla 8. Método de Tukey para la permeabilidad de vapor de agua (PVA) en películas de almidón de mandioca al 3\%. y 4\%

\begin{tabular}{lcccc}
\hline \multirow{2}{*}{ Tratamiento } & \multicolumn{2}{c}{ Almidón de mandioca al 3\% } & \multicolumn{2}{c}{ Almidón de mandioca al 4\% } \\
\cline { 2 - 5 } & $\begin{array}{c}\text { Permeabilidad } \\
\text { Promedio (\%) }\end{array}$ & $\begin{array}{c}\text { Tukey } \\
\boldsymbol{\alpha}=\mathbf{0 . 0 5}\end{array}$ & $\begin{array}{c}\text { Permeabilidad } \\
\text { Promedio (\%) }\end{array}$ & $\begin{array}{c}\text { Tukey } \\
\boldsymbol{\alpha}=\mathbf{0 . 0 5}\end{array}$ \\
\hline T3 & 0.00047 & $\mathrm{a}$ & 0.00036 & $\mathrm{~A}$ \\
$\mathrm{~T} 5$ & 0.00046 & $\mathrm{a}$ & 0.00037 & $\mathrm{~A}$ \\
T2 & 0.00043 & $\mathrm{a}$ & 0.00047 & $\mathrm{~A}$ \\
T1 & 0.00040 & $\mathrm{a}$ & 0.00045 & $\mathrm{~A}$ \\
T4 & 0.00036 & $\mathrm{a}$ & 0.00032 & $\mathrm{~A}$ \\
\hline
\end{tabular}


La prueba Tukey para el almidón de mandioca al 3\% indica que el tratamiento T3 es significativamente igual a todos los tratamientos.

La prueba Tukey para el almidón de mandioca al 4\% indicó que el tratamiento T2 es significativamente igual a todos los tratamientos.

Asimismo, se puede apreciar que el porcentaje de alargamiento o elongación en el almidón de banano verde oscila entre 2.47 y $1.65 \%$, siendo indicadas como el valor de mayor y menor elongación. Por otra parte, se puede observar que el porcentaje de elongación o alargamiento en el almidón de mandioca oscila entre 2.92 y $0.95 \%$, siendo indicadas como el valor de mayor y menor elongación.

Tabla 9. Propiedades mecánicas de almidón de banano verde.

\begin{tabular}{lccccc}
\hline Almidón (\%) & Muestra & Alargamiento (\%) & $\begin{array}{c}\text { Desviación } \\
\text { Estándar }\end{array}$ & ETC $\left(\mathbf{N} / \mathbf{m m}^{2}\right)$ & $\begin{array}{c}\text { Desviación } \\
\text { Estándar }\end{array}$ \\
\hline & 1 & 1.96 & 0.47 & 7.90 & 0.98 \\
A. Bv 3 \% & 2 & 1.65 & 0.47 & 8.33 & 1.78 \\
& 3 & 1.86 & 0.56 & 11.13 & 2.76 \\
& 4 & 2.27 & 0.75 & 7.15 & 1.85 \\
& 5 & 2.21 & 0 & 7.32 & 0.86 \\
\hline & 1 & 2.26 & $*$ & 20.23 & $*$ \\
& 2 & 2.09 & 0.06 & 9.01 & 0.78 \\
& 3 & 2.41 & 0.16 & 10.13 & 2.01 \\
& 4 & 2.47 & 0.53 & 10.33 & 0.68 \\
\hline
\end{tabular}

Nota. A.Bv: Almidón de banano verde / ETC: Esfuerzo de tracción en el corte /

*No muestra desviación estándar porque se tomó una sola repetición.

Tabla 10. Propiedades mecánicas del almidón de mandioca.

\begin{tabular}{lccccc}
\hline Almidón (\%) & Muestra & Alargamiento (\%) & $\begin{array}{c}\text { Desviación } \\
\text { Estándar }\end{array}$ & ETC (N/mm $\left.{ }^{2}\right)$ & $\begin{array}{c}\text { Desviación } \\
\text { Estándar }\end{array}$ \\
\hline & 1 & 1.36 & 0.35 & 2.31 & 1.63 \\
A. M 3\% & 2 & 2.12 & $*$ & 3.14 & $*$ \\
& 3 & 1.60 & 0.55 & 5.09 & 1.08 \\
& 4 & 2.57 & 0.29 & 6.45 & 1.39 \\
& 5 & 2.69 & 0.05 & 6.73 & 0.50 \\
\hline \multirow{2}{*}{ M 4\% } & 1 & 2.15 & 0.37 & 9.05 & 1.20 \\
& 2 & 0.95 & $*$ & 3.62 & $*$ \\
& 3 & 2.02 & 0.42 & 7.70 & 1.01 \\
& 4 & 2.84 & 0.16 & 9.19 & 0.29 \\
& 5 & 2.92 & 0.39 & 6.21 & 0.70 \\
\hline
\end{tabular}

Nota: A.M=Almidón de mandioca; ETC= Esfuerzo de tracción en el corte

*No muestra desviación estándar porque se tomó una sola repetición 
Tabla 11. Prueba de Tukey para el esfuerzo de tracción en el corte de películas de almidón mandioca al 3\%. Y $4 \%$

\begin{tabular}{lcccc}
\hline \multirow{2}{*}{ Tratamiento } & \multicolumn{2}{c}{ Almidón de mandioca al 3\% } & \multicolumn{2}{c}{ Almidón de mandioca al 4\% } \\
\cline { 2 - 5 } & ETC Promedio (\%) & $\begin{array}{c}\text { Tukey } \\
\mathbf{\alpha}=\mathbf{0 . 0 5}\end{array}$ & ETC Promedio (\%) & $\begin{array}{c}\text { Tukey } \\
\boldsymbol{\alpha}=\mathbf{0 . 0 5}\end{array}$ \\
\hline T5 & 6.73 & $\mathrm{a}$ & 6.21 & $\mathrm{~B}$ \\
$\mathrm{~T} 4$ & $\mathrm{a}$ & 9.20 & $\mathrm{~A}$ \\
$\mathrm{~T} 3$ & 6.45 & $\mathrm{ab}$ & 7.70 & $\mathrm{Ab}$ \\
$\mathrm{T} 2$ & 5.09 & $\mathrm{bc}$ & 3.62 & $\mathrm{C}$ \\
$\mathrm{T} 1$ & 3.14 & $\mathrm{c}$ & 9.05 & $\mathrm{~A}$ \\
\hline
\end{tabular}

La prueba Tukey para almidón de mandioca al 3\%indica que el tratamiento T5 es significativamente diferente a T2 y T1 pero significativamente igual a T4 y T3 ocupando el primer orden con esfuerzo de tracción en el corte promedio de $6.73 \%, 6.45 \%$ y $5.09 \%$ respectivamente; asimismo, se observa que el tratamiento T3 es significativamente diferente a T5, T4 y T1 pero no existe diferencia significativa con T2, ocupando estos el segundo orden. Por consiguiente, se aprecia que el tratamiento T2 es significativamente igual a T1, pero no a los tratamientos T5, T4 y T3 ocupando el tercer orden.

La prueba Tukey para almidón de mandioca al 4\% indicó que el tratamiento T4 es significativamente diferente a T5 y T2 pero significativamente igual a T1 y T3 ocupando el primer orden con solubilidad promedio de 9.20, 9.05 y $7.70 \%$ respectivamente; asimismo, se observa que el tratamiento T3 es significativamente diferente a T4, T1 y T2 pero no existe diferencia significativa en el T5, ocupando estos el segundo orden. Por consiguiente, se aprecia que T2 es significativamente diferente con todos los tratamientos.

Tabla 12. Método de Tukey para la elongación de películas de almidón de mandioca al $3 \%$. Y $4 \%$

\begin{tabular}{lcccc}
\hline \multirow{2}{*}{ Tratamiento } & \multicolumn{2}{c}{ Almidón de mandioca al 3\% } & \multicolumn{2}{c}{ Almidón de mandioca al 4\% } \\
\cline { 2 - 5 } & $\begin{array}{c}\text { Elongación } \\
\text { Promedio }(\%)\end{array}$ & $\begin{array}{c}\text { Tukey } \\
\mathbf{\alpha}=\mathbf{0 . 0 5}\end{array}$ & $\begin{array}{c}\text { Elongación } \\
\text { Promedio (\%) }\end{array}$ & $\begin{array}{c}\text { Tukey } \\
\boldsymbol{\alpha}=\mathbf{0 . 0 5}\end{array}$ \\
\hline T5 & 2.69 & $\mathrm{a}$ & 2.92 & $\mathrm{a}$ \\
$\mathrm{T} 4$ & 2.57 & $\mathrm{a}$ & 2.84 & $\mathrm{ab}$ \\
$\mathrm{T} 1$ & 1.36 & $\mathrm{c}$ & 2.15 & $\mathrm{bc}$ \\
$\mathrm{T} 3$ & 1.60 & $\mathrm{bc}$ & 2.02 & $\mathrm{C}$ \\
$\mathrm{T} 2$ & 2.12 & $\mathrm{ab}$ & 0.95 & $\mathrm{D}$ \\
\hline
\end{tabular}

La prueba Tukey para el almidón de mandioca al $3 \%$ indica que el tratamiento T5 es significativamente diferente a T3 y T1 pero significativamente igual a T4 y T2 ocupando el primer orden con elongación promedio de $2.69,2.57$ y $2.12 \%$ respectivamente; asimismo, se observa que el tratamiento T2 es significativamente diferente a T5, T4 y T1 pero no existe diferencia significativa en el tratamiento T3, 
ocupando estos el segundo orden. Por consiguiente, se aprecia que el tratamiento T3 es significativamente igual a T1 pero no a T5, T4 y T2 ocupando el tercer orden.

La prueba Tukey para almidón de mandioca al 4\% indica que el tratamiento T5 es significativamente diferente a T1, T3 y T2 pero significativamente igual a T4 ocupando el primer orden con elongación promedio de 2.92 y $2.84 \%$ respectivamente; asimismo, se observa que el tratamiento T4 es significativamente diferente a T5, T3 y T2 pero no existe diferencia significativa en el tratamiento T1, ocupando estos el segundo orden. Por consiguiente, se aprecia que el tratamiento T1 es significativamente igual a T3 y diferente a T5, T4 y T2. Por último, el tratamiento T2 es significativamente diferente con todos los tratamientos.

Los tratamientos en estudio de películas de almidón banano verde y mandioca son inversamente proporcionales, es decir, cuando el esfuerzo de tracción de corte es mayor el porcentaje de elongación disminuye.

\section{DISCUSIÓN}

Para la identificación de amilosa y amilopeptina, el grado de madurez del fruto es un factor importante para la obtención de mayores rendimientos en el almidón, ya que entre más inmaduro esté el fruto el contenido de almidón es mayor y diferente, porque a medida que avanza el proceso de maduración el almidón se convierte en azúcares (Badui, 1999). Similar manifestación lo ostentaron Abadía (1979) y Palmer (1979), quienes exponen que para aislar el almidón de plátano es necesario trabajar con la fruta en estado verde que es cuando posee la máxima cantidad de este (de 20 a $25 \%$ ). En la prueba de identificación de almidón según la USP NF 25, comprobó que los productos finales extraídos en la investigación definitivamente eran almidones y esto es debido a que la reacción química entre el almidón y la solución de yodo dando como resultado la formación de cadenas poliyoduro a partir de la reacción del almidón con el yodo presente en la Solución Lugol. Este color azul-negro desapareció al hidrolizarse o romperse el almidón en unidades estructurales menos pequeñas durante el calentamiento aplicado. Según Méndez (2010), la amilosa forma complejos de inclusión con yodo y es responsable del color azul característico del complejo almidón yodo. La amilopectina ha dado evidencias de no formar complejos estables con yodo, pero dan un color rojo pálido en su presencia.

El contenido de amilosa en el almidón de banano verde fue de $20.43 \%$ y de amilopectina $79.57 \%$. Kayisu et al. (1981) indican que el contenido de amilosa en el almidón de banano se encuentra entre 15 - $40 \%$. Para banano del grupo Cavendish, Kayisu (1981) reportó un contenido de 16\% en amilosa y Ling (1982) un contenido de 19.5\%. Para el almidón de banano del grupo Valery, García (1988) reportó un contenido de $17 \%$ y Waliszewski (2003) de $40.7 \%$ para la amilosa. Datos cercanos a lo analizado. 
Por otra parte, el contenido de amilosa en el almidón de mandioca fue de $14.36 \%$ y $85.64 \%$ de amilopectina, Repamar (2000) indica un contenido de amilosa en $16 \%$ y $84 \%$ de amilopectina en el almidón de yuca. Dato similar reporta Morín (2010) con un $17 \%$ de amilosa y $83 \%$ de amilopectina. Charles et al., (2005) reportaron que el contenido de amilosa en almidones de diferentes variedades de yuca (mandioca) fue de 15,9 a $22,4 \%$.

Del análisis químico del almidón, el contenido de lípidos encontrado en estudio de investigación osciló a $0.43 \%$ para la muestra de almidón de banano verde, Navarro (2016) reportó $0.20 \%$ de lípidos, menor a las obtenidas en las investigaciones de Zamudio-Flores en sus investigaciones del 2005 y 2008, donde obtuvo valores de $1.42 \%$ y $1.85 \%$ de lípidos, para almidón nativo de la misma variedad de fruto respectivamente.

El banano verde obtuvo el mayor porcentaje de humedad de $15.61 \%$ en comparación al almidón de mandioca de $9.53 \%$. Iles (2017) indica un menor contenido de humedad en el banano verde de $13.12 \%$, de igual modo lo indica Soto (2010) con un valor de $12.50 \%$. En cuanto al contenido de humedad en la mandioca, dato similar reportó Hernández et al., (2008) con 9.48 \% y Soto (2010) reporta un porcentaje de 8.50 menor a lo encontrado en esta investigación.

Respecto al contenido de cenizas para el almidón de banano verde se encontró el $0.26 \%$, dato menor a lo encontrado en la investigación de Iles (2017) quién indica 0.96 \% de cenizas presente en el banano verde. Para el almidón de mandioca se obtuvo el 0.07 \% cenizas, Morales (2014), reportó en el almidón de mandioca un contenido de cenizas de $0.24 \%$. Ballesteros (2011), indica un rango entre 0.50 a $1.50 \%$ de cenizas en el almidón de yuca y Hernández et al. (2008) reporta $0.29 \%$.

En cuanto a las películas formuladas a partir de banano verde, se registraron valores de contenido de humedad más altos cuando la concentración de almidón es menor, es decir, tienen mayor porcentaje de humedad las películas de almidón de banano verde al 3 \% y menor en las películas con una concentración de almidón de $4 \%$. Resultados similares a los obtenidos en esta investigación los encontraron Sánchez, García y Pinzón (2012) quienes manifestaron que se obtuvo una mayor humedad en las películas con una concentración de $3 \%$ con relación a las que contenían $4 \%$. Sanyang et al. (2015), manifestó que la concentración de almidón influye en el contenido de humedad de las películas.

Por otro lado, en las películas formuladas a partir de mandioca, las que presentaron mayor porcentaje de humedad fueron los tratamientos T4 y T5 del $4 \%$ de almidón. Ambos tienen en su composición quitosano-almidón.

Con respecto a la solubilidad, en el caso del banano verde al $3 \%$ el alto grado de solubilidad al agua en los tratamientos T4 y T5 las cuales tienen en su composición quitosano-almidón, diferente a los tratamientos T1, T2 y T3 que solo poseen almidón. Para el caso del banano verde al $4 \%$ por ser de mayor 
concentración se registró un alto grado de solubilidad en el T5, por lo tanto, se puede concluir que la concentración del almidón en las películas también influye la solubilidad al agua. Resultado respaldado por Navarro (2016) donde manifiesta que, a mayor concentración de almidón, mayor es la solubilidad en agua, concluyendo que la concentración de almidón en las películas influye en su capacidad de disolverse en agua.

Por otro lado, las películas de almidón de mandioca al 3\% registraron una mayor solubilidad en los tratamientos T1, T2 y T3, caso contrario ocurrió en los tratamientos T4 y T5 resultando ser menos solubles en agua. Esto indica que las películas de almidón de mandioca por si solas no necesitan de otro polímero para ser solubles. Igual resultado se obtuvo en el almidón de mandioca al $4 \%$.

Las películas elaboradas solamente con almidón poseen poca resistencia al agua, propiedades mecánicas y actividad antimicrobiana (Arifin et al., 2016). Unido con otro polímero resultan ser amigables proporcionando películas de baja solubilidad al agua.

El estudio en la permeabilidad al vapor de agua, se registró entre todas las películas al tratamiento T1 de almidón de banano verde $4 \%$ como el film de mayor permeabilidad con $0.00071 \mathrm{gmm} / \mathrm{hPam} 2$ y de menor permeabilidad al tratamiento T3 de almidón de banano verde $3 \%$ con $0.00034 \mathrm{gmm} / \mathrm{hPam} 2$, por consiguiente, se observa la relación con el espesor de las películas, ya que se registró un mayor espesor en el tratamiento T1 con 0.114 y un menor espesor en el tratamiento T3 con 0.064. Esto quiere decir que el espesor de las películas influye en la permeabilidad de los films. Similar análisis declaró Sobral (2001), quien observó que el aumento de la permeabilidad va en función al aumento del espesor. Declaración afín lo realiza Rutiaga (2002), en la cual declara que uno de los parámetros importantes para el desempeño de las propiedades de barrera de las películas es el espesor, ya que tiene una influencia directa en el desempeño de las mismas. Bertuzzi et al. (2007), a su vez también manifiestan que la permeabilidad es afectada generalmente por temperatura, espesura de la película, y el uso de un plastificante.

La humedad de las películas no influye en los valores de permeabilidad al vapor de agua, puesto que, de todas las películas, el tratamiento T2 de almidón de banano verde 3\% presenta mayor porcentaje de humedad con $4.79 \%$ y una permeabilidad al vapor de agua de $0.00058 \mathrm{gmm} / \mathrm{hPam} 2$. Contradiciendo a los resultados obtenidos por Valderrama (2014), los cuales indicaron que la capacidad de retención de agua disminuyó con el aumento de la humedad.

De las propiedades mecánicas identificadas, se registraron valores de elongación entre $0.95 \%$ a $2.84 \%$ entre todos los tratamientos, donde se pudo apreciar que, dentro de su parámetro, el porcentaje es bajo, esto es debido a la baja concentración de plastificante que se empleó para la formulación de las láminas. Navarro (2016), manifestó que dentro de sus evaluaciones el porcentaje oscila entre $9.17 \%$ a $14.37 \%$ donde el uso que le dio al plastificante fue sin dilución y más concentrado, obteniendo así un mayor 
porcentaje de elongación. Ello nos muestra la importancia del empleo de plastificantes para el producto que se requiera obtener. A mayor porcentaje de plastificante, mayor será su elongación y por consiguiente mejor esfuerzo de tensión en el corte. Sánchez-Aldana et al., (2015) apreció que a bajas concentraciones de glicerol la tensión fue mayor (Dos Reis et al., 2014) y citan que el incremento en la concentración de plastificante reduce las fuerzas intermoleculares con lo que se aumenta la flexibilidad y se reduce la resistencia de los materiales. La disminución de resistencia a la tensión de películas basadas en almidón por el aumento en la concentración del plastificante ha sido documentada por diversos autores (Días et al., 2010; Muscat et al., 2012; Jouki et al., 2013). Inicialmente las películas de biopolímero de almidón con plastificante son muy flexibles y luego de varios días se estabilizan y tornan más resistentes a la rotura por tracción manual (Arrieta y Palencia, 2016). Sánchez et al., (2012) manifiesta que el quitosano mejora levemente la tensión hasta la ruptura de las películas almidón - glicerol, aunque disminuye la flexibilidad de las mismas.

\section{CONCLUSIONES}

Ambos almidones, de banano verde y mandioca, fueron sometidos a análisis fundamentales, determinando que, el porcentaje de humedad y cenizas es mayor en el almidón de banano verde, a su vez que la presencia de lípidos es mínima en ambos casos.

Para las propiedades físicas, el espesor está directamente relacionados con la concentración de almidón. Asimismo, las películas que mostraron tener una mayor solubilidad al agua son aquellas que contienen almidón de mandioca (T1, T2 y T3), en el caso del almidón de banano verde se mostró mejor relación con el agua el T4 y T5. En cuanto a la permeabilidad al vapor de agua, el resultado influyó en la concentración de almidón y espesor de la película. De las propiedades mecánicas; se registró un mayor esfuerzo de tracción en el corte (ETC) en las películas elaboradas a partir de almidón de banano verde y menor esfuerzo de tensión en las películas de mandioca, haciéndolo a estas últimas menos rígidas.

En la biodegradación al suelo se estimó una pérdida importante de peso de las películas, mostrando así, que estos se pueden degradar en un tiempo mucho menor al usual de los plásticos convencionales. Las películas que registraron mayor pérdida de peso fueron aquellas cuya mezcla tuvo quitosano-almidón.

\section{REFERENCIAS BIBLIOGRÁFICAS}

Abadía, P. (1979). Producción de almidón de banano por el método enzimático. Proyecto de graduación. Lic. Ing. Química. San José, Costa Rica, Universidad. Facultad de Ingeniería. 89 p. American Society for Testing and Material. 2000. Standard terminology relating to plastics. ASTM D883 International. 
Alves, V. D., Mali, S., Beléia, A., y Grossmann, M. (2007). Effect of glycerol. Journal of Food Engineering, 941-946.

Arifin, Budi; Sugita, Purwantiningsih and Masyudi, Dery Ermawan. (2016). Chitosan and lauric acid addition to corn starch-film based effect: physical properties and antimicrobial activity study. International Journal of Chemical Sciences 14(2):529-544.

Arrieta, A. y Palencias, S. (2016). Estudio electroquímico de un biopolímero compuesto PPy/almidón de cassava. Revista Latinoamericana de Metalurgia y Materiales. 36(1):26-35.

Ballesteros. (2011). Composición de raíces de mandioca y tubérculos de camote. Pag. 12.

Badui, D. (1999). Química de los alimentos. Cuarta Edición. Editorial Pearson. México. Capítulo 1.

Bertuzzi, M. A., Armada, M., and Gottifredi, J. C. (2007). Physicochemical characterization of starchbased films. Journal of Food Engineering. 82(1): 17-25.

Castillo, R., Escobar, E., y Fernández, D. (2015). Biopástico a base de cáscara de plátano. Revista de iniciación científica - RIC, 34-37.

Charles, A. et al. (2005). Influence of amylopectin structure and amylose content on the gelling properties of five cultivars of cassava starches. Journal of Agricultural and Food Chemistry, v. 53, n. 7, p. 2717-2725.

Dos Reis, R. C., Devilla, I. A., Oliveira, G. H. H., Côrrea, P. C., Ascheri, D. P., Souza, A. B. y Servulo, A. C. O. (2014). Propriedades mecánicas, permeabilidad y solubilidad de la película biodegradable de almidón de ñame y glicerol. Interciencia, 39(6), 410-415.

Días, B., Carmen, M., Fábio, D. y João, B. (2010). Biodegradable films based on rice starch and rice flour. Journal of Cereal Science. 51(2):213-219.

García, E. y Lajolo, F. (1988). Starch transformation during banana ripening: The amylase and glucosidase behavior. Journal of Food Science. Pag. 1181-1186.

Hernández, A., y Vásquez, L. (2008). Optimización del sistema mixto de secado de yuca (manihotesculenta crantz.) instalado en la planta agroindustrial de Morroa para corporación Minuto de Dios. Sincelejo. Colombia. Universidad de Sucre

Iless, S. (2017). Estudio de materiales termoplásticos obtenidos a partir de un copoliéster alifático aromático y almidón de banano (Musa paradisiaca). Pag. 32

Jouki, M., Tabatabaei Yazdi, F., Mortazavi, S.A. y Koocheki, A. (2013). Physical, barrier and antioxidant properties of a novel plasticized edible film from quince seed mucilage. International Journal of Biological Macromolecules 62, 500-507.

Kayisu, K. y Hood, L. (1981). Molecular structure of banana starch. Journal of Food Science. Pag. 18941897. 
Ling, L., Osman, E., Fernández, J. y Reilly, P. (1982). Physical properties of starch from Cavendish banana fruit. Starch/Staerke. Pag. 184-188.

Méndez, A. (2010). Extracción de almidón de banano verde (Musa sapientum variedad Cavendish) producto de desecho de las industrias bananeras y evaluación de su función como excipiente en la formulación de comprimidos. (tesis de licenciatura). Universidad de San Carlos de Guatemala, Facultad de Ciencias Químicas y Farmacia. Guatemala.

Morales, E. (2014). Caracterización de películas elaboradas a partir de harina de quinua (Chenopodium quinoa, Willd), almidón modificado de mandioca (Manihot esculenta) y montmotillonita. Escuela Politécnica Nacional. Facultad de Ingeniería Química y Agroindustrial - Quito.

Morín, E. (2010). Elaboración y caracterización de películas comestibles a base de almidón de maíz ceroso y mucílago de nopal (opuntia ficus) usando la tecnología de extrusión termoplástica. Universidad Autónoma de Querétaro (México). Maestría en Ciencia y Tecnología de Alimentos.

Muscat D, Adhikari B, Adhikari R, Chaudhary DS (2012) Comparative study of film forming behaviour of low and high amylose starches using glycerol and xylitol as plasticizers. J. Food Eng. 109: 189-201.

Navarro, M. (2016). Influencia de la fuente y concentración de almidón sobre las propiedades físicoquímicas y estructurales de las películas quitosano-almidón combinadas con glicerol para su uso en la agroindustria. Pag. 51

Palmer, J. (1979). Banano Products. In tropical foods chemistry and nutrition. Inglaterra. V.2. pp 625635.

Pinzón, M. I., García, O. R. Sánchez, L.T. (2012). Elaboración y caracterización de películas de almidón de yuca (Manihot esculenta) variedad Ica cultivada en el departamento del Quindío. Vitae, v.19 fasc. 1 p.S426 - S429.

Repamar, M., Cepis, P.S. (2000). Impacto ambiental de productos químicos auxiliares usados en la industria textil Argentina. Productos de acabados.

Rutiaga, Q. (2002). Elaboración de películas plásticas flexibles a partir de polímeros naturales como una alternativa de empaque y la evaluación de sus propiedades. Universidad Autónoma de Nuevo Leon - Facultad de Ciencias Biológicas. México.

Sánchez-Aldana, D., Contreras-Esquivel, J., Nevárez-Moorillón, G. y Aguilar, C. (2015). Caracterización de películas comestibles a base de extractos pécticos y aceite esencial de limón mexicano. CyTA - Journal of Food. 13(1):17-25. 
Sánchez, T., García, O. y Pinzón, M. (2012). Elaboración y caracterización de películas de almidón de mandioca (manihot esculenta) variedad Ica cultivada en el departamento de Quindío. Vitae, 19 (1):S426-S429.

Sanyang. M., Sapuan, S., Jawaid, M., Ishak, M. y Sahari, J. (2015). Effect of glycerol and sorbitol plasticizers on physical and thermal properties of sugar palm starch based films. In Recent Advances in Environment, Ecosystems and Development. Proceedings of the 13th International Conference on Environment, Ecosystems and Development. April 23-25. (pp. 157-162). Kuala Lumpur, Malaysia.

Sobral, P. D. A., Menegalli, F. C., Hubinger, M. D., y Roques, M. A. (2001). Mechanical, water vapor barrier and thermal properties of gelatin based edible films. Food hydrocolloids, 15(4-6), 423432.

Soto, V. (2010). Cuantificación de almidón total y de almidón resistente en harina de plátano verde (musa cavendishii) y banana verde (musa paradisíaca). Revista Boliviana de Química, 27(2), 94-93. $\begin{array}{lllllll}\text { Recuperado en } & \text { de } & \text { junio }\end{array}$ http://www.scielo.org.bo/scielo.php?script=sci_arttext\&pid=S0250-

$54602010000200004 \& \operatorname{lng}=$ es\&tlng=es.

Valderrama, B. (2014). Evaluación del comportamiento de películas comestibles de quitosano almacenadas bajo condiciones controladas. Universidad Nacional de Colombia. Facultad de Ciencias Agrarias Bogotá - Colombia.

Vázquez, A., Beltrán, M., Espinosa, R. M. y Velasco, M. (2018). El origen de los plásticos y su impacto en el ambiente. Obtenido de https://anipac.com/wpcontent/uploads/2018/09/origendelosplasticos.pdf

Waliszewski, K., Aparicio, M., Bello, L. y Monroy, J. (2003). Changes of banana starch by chemical and physical modification. Carbohydrate Polymers. Pag. 237-242.

Zamudio-Flores, P. (2005). Elaboración de películas degradables de almidón de plátano: evaluación de sus propiedades mecánicas y de barrera. Tesis de Maestría. Centro de Desarrollo de Productos Bióticos del Instituto Politécnico Nacional. Yautepec, Morelos, México.

Zamudio-Flores, P. (2008). Caracterización estructural de películas elaboradas con almidón modificado de plátano y con quitosano. Tesis de Doctorado. Centro de Desarrollo de Productos Bióticos del Instituto Politécnico Nacional. Yautepec, Morelos, México 